hep-th/9904003

LPTENS 99/09

ULB-TH/99-07

\title{
Noether superpotentials in supergravities
}

\author{
M. Henneaux ${ }^{a, b}$, B. Julia ${ }^{c}$ and S. Silva ${ }^{c}$ \\ ${ }^{a}$ Physique Théorique et Mathématique, Université Libre de Bruxelles, \\ Campus Plaine C.P. 231, B-1050 Bruxelles, Belgium \\ ${ }^{b}$ Centro de Estudios Científicos de Santiago, \\ Casilla 16443, Santiago 9, Chile \\ ${ }^{c}$ Laboratoire de Physique Théorique CNRS-ENS \\ 24 rue Lhomond, F-75231 Paris Cedex 05, France円
}

\begin{abstract}
Straightforward application of the standard Noether method in supergravity theories yields an incorrect superpotential for local supersymmetry transformations, which gives only half of the correct supercharge. We show how to derive the correct superpotential through Lagrangian methods, by applying a criterion proposed recently by one of us. We verify the equivalence with the Hamiltonian formalism. It is also indicated why the first-order and second-order formalisms lead to the same superpotential. We rederive in particular the central extension by the magnetic charge of the $\mathcal{N}_{4}=2$ algebra of SUGRA asymptotic charges.
\end{abstract}

\footnotetext{
${ }^{1}$ Unité propre du CNRS, associée à l'Ecole Normale Supérieure et à l'Université de Paris-Sud. This work has been partly supported by the EU TMR contract ERBFMRXCT96-0012.
} 


\section{Introduction}

The problem of defining meaningful conserved charges in gauge theories is notoriously subtle. It has been addressed in the literature along various lines. One approach relies on the use of Noether identities and conserved currents [1, 2, 3, 4, 5, 6, 7]. Another is based on Hamiltonian methods and asymptotic symmetries [8, 9, 10, 11. The first approach is probably the most familiar and emphasizes locality. It has been recognized, however, that it suffers from ambiguities, which, if improperly resolved, may lead to incorrect results. It was recalled in [7] that in modern language, Noether already showed that onshell the conserved charge associated to one-parameter subgroup of a gauge group is topological and hence lives at any infinity (ignoring singularities). More than 40 years later 8 , 9], it was understood that charges can indeed be defined at infinity for a good choice of boundary conditions, and in one to one correspondence with their (asymptotic) symmetries. The later may be finite or infinite in number.

The purpose of this paper is to derive the correct superpotential for the supercharges in supergravity theories. We show that a "natural" application of the Noether identities yields an incorrect supercharge. We then derive a correct superpotential by adopting the criterion proposed in 12] and verify equivalence with the Hamiltonian approach. Finally, we explain in a first appendix why the first-order and second-order formulations yield the same superpotentials for local supersymmetries. The second appendix analyses in some detail the case of $\mathcal{N}_{4}=2$ supergravity and provides explicit boundary conditions for the fields that enable one to meaningfully compute the charges and their algebra that contain a central charge as in the rigid supersymmetry case.

\section{Noether superpotential}

As shown by E. Noether, any continuous one-parameter invariance of the action leads to a conservation law $\partial_{\mu} j^{\mu} \approx 0$, where $\approx$ means "equal to when the equations of motion hold". The conserved Noether current is defined through

$$
j^{\mu}=S^{\mu}-\frac{\partial L}{\partial \partial_{\mu} \phi} \delta \phi
$$

where $\delta \phi$ is the infinitesimal variation under the (local or global) symmetry and $\delta L=\partial_{\mu} S^{\mu}$. The fields $\phi$ may carry an index over which one sums in 
(2.1), but this will not be explicitly indicated. In the class of Lagrangians having the same (bulk) Euler-Lagrange derivative $\left(L \rightarrow L+\partial_{\mu} k^{\mu}\right)$, one must adjust the surface term in such a way that the action $\int d^{D} x L=0$ is truly stationary on-shell. This surface term is fixed by a choice of boundary conditions.

However, even for a given $L$, there is some ambiguity in the choice of $S^{\mu}$ since the addition to $S^{\mu}$ of the divergence $\partial_{\nu} k^{\mu \nu}$ of an antisymmetric tensor $k^{\mu \nu}=-k^{\nu \mu}$ does not modify $\partial_{\mu} S^{\mu}$. Expressed in terms of the currents, this (topological) ambiguity reads

$$
j^{\mu} \rightarrow j^{\mu}+\partial_{\nu} k^{\mu \nu}
$$

and is particularly relevant in the case of gauge symmetries.

Indeed, in this case, conserved Noether currents $j^{\mu}$ derive from superpotentials,

$$
j^{\mu} \approx \partial_{\nu} U^{\mu \nu}, U^{\mu \nu}=-U^{\nu \mu}
$$

This has been proved in many references (see [1, 2, 3, 4, 5, 6, [7] and also [13] for a cohomological interpretation). The fact that $j^{\mu}$ derives from a superpotential implies that one can set it equal to zero by means of the redefinitions (2.2). In other words, "everything is in the superpotential", which indicates how crucial it is to resolve correctly the above-mentioned ambiguities. A wrong choice would lead to an incorrect integrated charge,

$$
Q=\int_{V} d^{D-1} x j^{0} \approx \int_{\partial V} d^{D-2} S_{i} U^{0 i}
$$

which would e.g. not generate the appropriate transformations through the Poisson bracket.

A "natural" choice for the superpotential may seem to be

$$
U^{\mu \nu}=-\frac{1}{2}\left(M_{A}^{\mu \nu}-M_{A}^{\nu \mu}\right) \xi^{A}
$$

with

$$
M_{A}^{\mu \nu}=\frac{\partial L}{\partial \partial_{\mu} \phi} \Delta_{A}^{\nu}
$$

One finds

$$
j^{\mu}=\partial_{\nu} U^{\mu \nu}+\frac{\delta L}{\delta \phi} \Delta_{A}^{\mu} \xi^{A}
$$

where the $\Delta_{A}^{\mu}$ 's are the coefficients of the derivatives of the gauge parameters in the variations of the fields,

$$
\delta_{\xi} \phi=\xi^{A} \Delta_{A}+\partial_{\mu} \xi^{A} \Delta_{A}^{\mu}
$$


(we assume for simplicity that only the first-order derivatives appear). The choice (2.5) corresponds to taking the coefficient of $\partial_{\nu} \xi^{A}$ in $S^{\mu}=\xi^{A} \Sigma_{A}^{\mu}+$ $\partial_{\nu} \xi^{A} \Sigma_{A}^{\mu \nu}$ to be symmetric in $\mu, \nu$ and may be regarded as being "natural" on this ground.

However, this choice is not always correct and does in fact give an incorrect supercharge in supergravity for instance.

To see this, note that only the fields that transform into derivatives of the gauge parameter contribute to the superpotential, and only the piece of the action containing derivatives of these fields is relevant. In all supergravity theories, the relevant part of the supersymmetry transformations is thus

$$
\delta_{\epsilon} \psi_{\sigma}^{A}=\partial_{\sigma} \epsilon^{A}+\text { "more" }
$$

and the relevant piece in the action is the kinetic term for the gravitini?,

$$
\frac{i}{2} \bar{\psi}_{\lambda}^{A} \gamma^{\lambda \mu \nu} \partial_{\mu} \psi_{\nu}^{A}
$$

One finds by application of formulas 2.5 2.6

$$
U_{\bar{\epsilon}}^{\mu \nu}=-\frac{i}{2} \bar{\epsilon}^{A} \gamma^{\mu \nu \lambda} \psi_{\lambda}^{A}
$$

As discussed in the first appendix, one obtains (2.11) by working either in first or second order formalism.

Although simple, the formula (2.11) is incorrect. It gives only half of the supercharge as can be seen by comparing with the Hamiltonian formalism [14] or by computing the variation of the supercharge under a supersymmetry transformation, where one finds only half of the 4-momentum $P^{\mu}$ instead of $P^{\mu}$ itself. The correct supercharge is [14]

$$
Q_{\bar{\epsilon}}=-i \int_{S_{\infty}} d^{D-2} S_{i} \bar{\epsilon}^{A} \gamma^{0 i k} \psi_{k}^{A}
$$

while the integral of $j^{0}$ given by (2.7), (2.11) is clearly only half of this expression. That (2.11) is incorrect is perhaps not surprising since it is well appreciated that there exist in general relativity a plethora of superpotentials, many of which yield incorrect energy, momentum, or angular momentum [4, 15]. For a recent and informative discussion, see [16]. We

\footnotetext{
${ }^{2}$ The conventions are the following: $\eta^{a b}=\{-,+, \ldots,+\}, \epsilon_{01 \ldots(D-1)}=1, \gamma^{a}$ are $D$ real Majorana matrices and $\gamma^{a_{1} \ldots a_{i}}:=\gamma^{\left[a_{1}\right.} \ldots \gamma^{\left.a_{i}\right]}$. The gravitini are described by Majorana spinors.
} 
have just pointed out the supersymmetric extension of this problem. What is needed is a criterion that selects among the many candidate superpotentials the correct one. Such a criterion has been proposed in [12] and tested with success in many models. We apply below this criterion and show that it yields the correct supercharge.

\section{Construction of the correct surface integrals at infinity}

The approach proposed in [12] is a "superpotential-based generalization" of the Hamiltonian approach of [9]. It may not always be equivalent to it, but in the case of theories like supergravity where one can write the Lagrangian in terms of forms and exterior products (in the sense specified after equation (3.4)), it does yield the same supercharge.

The starting point of [12] is the relationship between the superpotential and the conserved current associated with a given one parameter group of gauge transformations of the fields through the Noether identities. For any choice of surface terms, this relationship reads [7]

$$
j_{U}^{\mu}=\partial_{\nu} U^{\mu \nu}+\frac{\delta L}{\delta \phi} \Delta_{A}^{\mu} \xi^{A}
$$

with the term proportional to the equations of motion being independent of the choice. The idea is then to find a criterion which, given the term proportional to the equations of motion, completes it in a definite way in equation (3.1) ).

In the case of supergravity, the supersymmetry current identity is

$$
\begin{aligned}
j_{\bar{\epsilon}}^{\mu} & =\partial_{\nu} U_{\bar{\epsilon}}^{\mu \nu}+\bar{\epsilon}^{A} \frac{\delta L}{\delta \bar{\psi}_{\mu}^{A}} \\
& =\partial_{\nu} U_{\bar{\epsilon}}^{\mu \nu}+i \bar{\epsilon}^{A} \gamma^{\mu \rho \sigma}\left(\partial_{\rho} \psi_{\sigma}^{A}+\Lambda_{\rho \sigma}^{A}\right)
\end{aligned}
$$

where $\Lambda_{\rho \sigma}^{A}$ denotes terms containing undifferentiated gravitini fields.

If one varies this equation with respect to the gravitini fields, one gets,

\footnotetext{
${ }^{3}$ The current in (3.1) is conserved on-shell due to the antisymmetry of $U^{\mu \nu}$ and the so-called Noether identities, $\partial_{\mu}\left(\frac{\delta L}{\delta \phi} \Delta_{A}^{\mu} \xi^{A}\right)=\frac{\delta L}{\delta \phi} \delta_{\xi} \varphi$.

${ }^{4}$ In computing the superpotential associated with supersymetries, one may assume $\delta$ (other fields) $=0$, since the terms proportional to $\delta$ (other fields) die off faster at infinity, where the superpotential is actually defined. For instance, in four dimensions, this condi-
} 
upon integration by parts,

$$
\delta j_{\bar{\epsilon}}^{\mu}=\partial_{\nu} \delta U_{\bar{\epsilon}}^{\mu \nu}+\partial_{\nu} V^{\mu \nu}-i \partial_{\nu} \bar{\epsilon}^{A} \gamma^{\mu \nu \sigma} \delta \psi_{\sigma}^{A}+i \bar{\epsilon}^{A} \gamma^{\mu \rho \sigma} \delta \Lambda_{\rho \sigma}^{A}
$$

with

$$
V^{\mu \nu}(\epsilon, \phi, \partial \phi)=i \bar{\epsilon}^{A} \gamma^{\mu \nu \sigma} \delta \psi_{\sigma}^{A}
$$

Note that here $V^{\mu \nu}$ is antisymmetric in $\mu$ and $\nu$. In fact, $V^{\mu \nu}$ is defined by (3.3) up to a total divergence, $\tilde{V}^{\mu \nu}=V^{\mu \nu}+\partial_{\rho} X^{\mu[\nu \rho]}$, which can in the present case be adjusted so that $V^{\mu \nu}$ is antisymmetric in $\mu$ and $\nu$. Quite generally, the antisymmetry is guaranteed if the theory can be written in a first order formulation (see appendix of [12]).

What is proposed in [12] is to take $U^{\mu \nu}$ in such a way that the divergence terms cancel in (3.3), i.e., such that

$$
\delta U_{\bar{\epsilon}}^{\mu \nu}+V^{\mu \nu}=0
$$

The integration in field space of this equation is straightforward and leads to

$$
U_{\bar{\epsilon}}^{\mu \nu}=-i \bar{\epsilon}^{A} \gamma^{\mu \nu \sigma} \psi_{\sigma}^{A}
$$

without the factor one-half (recall that $\delta$ (other fields) $=0$ - as stated in [12], (3.5) must be imposed only at infinity). As in the Hamiltonian formalism of [9], equation (3.5) defines the charge up to a constant which can be adjusted so that the superpotential vanishes for the vacuum. This superpotential is correct since, contrary to (2.11), it yields the correct supercharges (2.12). The last two terms of (3.2) are not relevant for charge evaluation. Actually the bulk charge has not yet been defined for singular solutions even in the presence of horizons.

That the supercharge that follows from (3.5) is the same as the one obtainable by Hamiltonian methods is easy to understand. Indeed, one may identify $\partial_{i} V^{0 i}$ as the surface term that one picks up at the spatial boundary when breaking the variation of the spatial integral of the field-equation term in $(3.1)$

$$
\delta \int_{x^{0}=C^{t}} d^{D-1} x \frac{\delta L}{\delta \phi} \Delta_{A}^{0} \xi^{A}:=\delta \int_{x^{0}=C^{t}} d^{D-1} x \bar{\epsilon}^{A} \frac{\delta L}{\delta \bar{\psi}_{0}^{A}}
$$

tion is verified in the asymptotically flat case, for which we adopt the boundary conditions of appendix B, or in the asymptotically anti-de Sitter case, for which we take the precise boundary conditions of [17]. Thus the expression (3.6) for the superpotential associated with supersymmetries is correct in both cases. 
into a bulk term and a surface term,

$$
\delta \int_{x^{0}=C^{t}} d^{D-1} x \bar{\epsilon}^{A} \frac{\delta L}{\delta \bar{\psi}_{0}^{A}}=\text { "bulk" }+\int_{S_{\infty}} d^{D-2} S_{i} V^{0 i} .
$$

where "bulk" contains only undifferentiated variations of the canonical fields. This identification manifestly holds in supergravity. The only field equation that appears in (3.8) is the equation associated with the field that transforms into the time derivative of the gauge parameter, i.e. $\bar{\psi}_{0}^{A}$.

Now, it is well-known that the temporal component $\bar{\psi}_{0}^{A}$ of the gravitino field is the Lagrange multiplier for the supersymmety constraint-generator, namely $\frac{\delta L}{\delta \psi_{0}^{A}}$. Therefore, the term being varied in (3.8) is of the form "Lagrange multipliers" times "Hamiltonian constraints". This property, verified here for supergravity, is actually generic since the Lagrange multipliers transform always into the time derivatives of the gauge parameters (see e.g. [18]). Thus, the zeroth component of the criterion (3.5)

$$
\delta U_{\bar{\epsilon}}^{0 i}+V^{0 i}=0
$$

precisely guarantees that the charge

$$
Q_{\bar{\epsilon}}=\int_{x^{0}=C^{t}} d^{D-1} x \bar{\epsilon}^{A} \frac{\delta L}{\delta \bar{\psi}_{0}^{A}}+\int_{S_{\infty}} d^{D-2} S_{i} U_{\bar{\epsilon}}^{0 i}
$$

has well-defined functional derivatives in the sense of [9], i.e., has a variation that contains only a bulk part. Since the asymptotic equality $\delta U_{\bar{\epsilon}}^{0 i}+V^{0 i}=0$, together with a covariance argument at infinity, implies $\delta U_{\bar{\epsilon}}^{\mu \nu}+V^{\mu \nu}=0$ (asymptotically), one may conclude that in the case of supergravity, the superpotential method supplemented by the criterion of [12] and the Hamiltonian method are equivalent.

\section{Conclusions}

In this paper, we have shown how superpotential methods apply to local supersymmetry transformations in supergravity. We have rederived the correct supercharges. Once the supercharges are known, one may compute their algebra [14, 19]. The case of $\mathcal{N}_{4}=2$ supergravity is particularly interesting because the algebra of the supercharges contains central charges. One of these, the magnetic central charge, arises in exactly the same way as the conformal central charge in $2+1$ anti-de Sitter gravity [20]: the central charge is not seen in the algebra of the asymptotic symmetries, but 
does appear in the algebra of their canonical generators. The calculation is direct. Because it lies somewhat outside the main line of this paper, it is discussed in the second appendix where precise boundary conditions that include magnetic sources are displayed.

\section{Acknowledgements}

We thank Eugène Cremmer and Thomas Materna for useful conversations. MH is grateful to the "Laboratoire de Physique Théorique de l'Ecole Normale Supérieure" for warm hospitality extended to him in May of 1998 while this work began. The work of $\mathrm{MH}$ has been partly supported by the "Actions de Recherche Concertées" of the "Direction de la Recherche Scientifique - Communauté Française de Belgique", by IISN - Belgium (convention 4.4505.86) and by Proyectos FONDECYT 1970151 and 7960001 (Chile).

This work has been partly supported by the EU TMR contract ERBFMRXCT960012 .

\section{Appendix A: Superpotential in first-order and second- order formalisms}

The superpotentials for pure gravity are better understood in a pure $1^{\text {st }}$ order formalism, i.e. where the connection $\omega_{\mu b}^{a}$ is varied independently [7]. However, supergravities are usually given in a $2^{\text {nd }}$-order formulation of the supersymmetry transformations laws. That is the (spin)-connection is not treated as an independent field, and then, its supersymmetry transformation law is not required. The point of this appendix is to check in the special case of $\mathcal{N}_{4}=2$ supergravity that the above computation of the superpotential does not depend on which formalism $\left(1^{\text {st }}\right.$ or $2^{\text {nd }}$ order) we are using. This

provides a consistency check for [12]. The same consistency holds for pure gravity [21].

First of all, the starting point for computing superpotentials is equation (3.1). In the special case of supergravity, the result was given in (3.2). Now, the only difference between a $1^{\text {st }}$ or $2^{\text {nd }}$ order computation would come from a term proportional to the equation of motion of the connection when computing $V^{\mu \nu}$ using the second term of the rhs of equation (3.1). However, if the $1^{\text {st }}$ order formulation of supergravity is such that the supersymmetry 
variation of the connection does not contain any term proportional to $\partial_{\mu} \xi^{A}$, no additional contribution is expected (recall the definitions (2.8) and (3.1)).

In a parallel publication [22], we will present a general scheme to derive a pure $1^{\text {st }}$ order formulation from a 1.5 one. That simply requires to compute a supersymmetry transformation law for the connection. Then, the simplest way to show that the superpotential computation for $1^{\text {st }}$ order formulation is equivalent to the $2^{\text {nd }}$ order one is to show that this supersymmetry transformation law does not depend on the derivative of the gauge parameter. This holds for the $\mathcal{N}_{4}=1$ supergravity, where the supersymmetry transformation law for the connection is 23:

$$
\delta_{1} \omega_{\mu}^{a b}=2 i \kappa^{2} \bar{\epsilon} \gamma^{5}\left(\gamma_{\mu} \tilde{\psi}^{a b}-\frac{1}{2} e_{\mu}^{a} \gamma_{c} \tilde{\psi}^{c b}+\frac{1}{2} e_{\mu}^{b} \gamma_{c} \tilde{\psi}^{c a}\right)
$$

where $\tilde{\psi}^{a b}:=\frac{1}{2} \epsilon^{a b c d} \mathcal{D}_{c} \psi_{d}$, with the covariant derivative acting on spinors defined as usual by $\mathcal{D}_{\sigma}:=\partial_{\sigma}+\frac{\gamma_{a b}}{4} \omega_{\sigma}^{a b}$.

In the $\mathcal{N}_{4}=2$ supergravity case, it is also possible to find a $1^{\text {st }}$ order formulation, that is a supersymmetry transformation law for the connection. The result is [22]:

$$
\begin{gathered}
\delta_{1} \omega_{\mu}^{a b}=2 i \kappa^{2} \bar{\epsilon}^{A} \gamma^{5}\left(\gamma_{\mu} \tilde{\hat{\psi}}_{A}^{a b}-\frac{1}{2} e_{\mu}^{a} \gamma_{c} \tilde{\hat{\psi}}_{A}^{c b}+\frac{1}{2} e_{\mu}^{b} \gamma_{c} \tilde{\hat{\psi}}_{A}^{c a}\right) \\
+i \kappa^{3} \bar{\epsilon}_{A} \varepsilon_{B}^{A}\left(\hat{F}^{a b}-\tilde{\hat{F}}^{a b} \gamma^{5}\right) \psi_{\mu}^{B}
\end{gathered}
$$

Here $\tilde{\hat{\psi}}_{a b}^{A}:=\frac{1}{2} \varepsilon_{a b c d} \hat{\mathcal{D}}^{c} \psi^{d A}$. The $\left(\mathcal{N}_{4}=2\right)$ - "hatted" covariant derivative is defined by $\hat{\mathcal{D}}_{\sigma}:=\mathcal{D}_{\sigma}+\frac{\kappa}{2} \varepsilon_{B}^{A}\left(\hat{F}_{\rho \sigma} \gamma^{\rho}+\tilde{\hat{F}}_{\rho \sigma} \gamma^{\rho} \gamma^{5}\right)$ together with (supercovariant) "hatted" field strength $\hat{F}_{\mu \nu}:=F_{\mu \nu}+i \kappa \varepsilon_{A B} \bar{\psi}_{\mu}^{A} \psi_{\nu}^{B}$ and its Hodgedual $\tilde{\hat{F}}_{\mu \nu}:=\frac{1}{2} \varepsilon_{\mu \nu \rho \sigma} \hat{F}^{\rho \sigma}$.

Again, there is no term in $\partial_{\mu} \bar{\epsilon}^{A}$ in the above transformation law. Another example is the eleven dimensional supergravity, which will be presented also in [22]. 


\section{Appendix B: Boundary conditions, asymptotic sym- metry algebra and algebra of charges for $\mathcal{N}_{4}=2$ supergravity}

To compute the algebra of the charges of $\mathcal{N}_{4}=2$ supergravity, one first needs to give boundary conditions for the fields at spatial infinity that ensure that the surface integrals yielding the corresponding charges be all finite. The boundary conditions should also be invariant under the asymptotic symmetries, i.e., under the asymptotic rigid $N=2$ SUSY algebra. In the second order formalism, the independent fields of $\mathcal{N}_{4}=2$ supergravity are the tetrads $e_{\mu}^{a}$, the abelian connection $A_{\mu}$ and the two gravitini $\psi_{\mu}^{A}$. The boundary conditions for the tetrads and the gravitini are given in [9, 14] (the tetrads approach their Minkowskian values up to terms falling off like $1 / r$ with definite parity conditions, while the gravitini fall off like $1 / r^{2}$ ). For this reason, we shall focus here only on the abelian connection. Our treatment includes magnetic monopoles.

As in [9, 14], we shall express the boundary conditions in terms of the canonical variables, which are $A_{k}$ and $\pi^{k}$ (electric field). To enforce the Lorentz invariance properties of the boundary conditions, it is convenient to use the "improved" form of the transformation of the connection under diffeomorphisms, which is

$$
\delta_{\xi} A_{\mu}=\xi^{\nu} F_{\nu \mu}
$$

This is in fact the transformation that arises in the Hamiltonian formalism [24. Indeed, the constraints associated with diffeomorphisms are, for $N=2$ supergravity,

$$
\begin{aligned}
\mathcal{H} & =\mathcal{H}^{G}+\mathcal{H}^{e m}+\mathcal{H}^{\frac{3}{2}} \approx 0, \\
\mathcal{H}_{k} & =\mathcal{H}_{k}^{G}+\mathcal{H}_{k}^{e m}+\mathcal{H}_{k}^{\frac{3}{2}} \approx 0
\end{aligned}
$$

where $\mathcal{H}^{G}$ and $\mathcal{H}_{k}^{G}$ are the metric contributions, while $\mathcal{H}^{\text {em }}$ and $\mathcal{H}_{k}^{e m}$ (re-

spectively, $\mathcal{H}^{\frac{3}{2}}$ and $\mathcal{H}_{k}^{\frac{3}{2}}$ ) are the electromagnetic contributions (respectively, the gravitini contributions). We shall only need here the electromagnetic terms (the gravitational terms do not contribute to the transformations of the electromagnetic variables, while the spin $3 / 2$ terms can be neglected asymptotically). One has

$$
\begin{aligned}
\mathcal{H}^{e m} & =\frac{g_{i j}}{2 \sqrt{g}}\left(\pi^{i} \pi^{j}+\mathcal{B}^{i} \mathcal{B}^{j}\right) \\
\mathcal{H}_{k}^{e m} & =F_{k m} \pi^{m}
\end{aligned}
$$


(electromagnetic energy density and Poynting vector). Here, $\mathcal{B}^{i}$ is the magnetic field and $g_{i j}$ the spatial metric induced on the surfaces $x^{0}=C^{t} ; g$ is its determinant. It is straightforward to check that $\int d^{3} x\left(\xi \mathcal{H}+\xi^{k} \mathcal{H}_{k}\right)$, where $\xi$ is the displacement normal to the hypersurface $x^{0}=C^{t}$ and $\xi^{k}$ the tangential displacement, generates (B.1) when acting on $A_{k}$ through the Poisson bracket. The transformation law (B.1) for $A_{0}$ follows then from the general variation of the Lagrange multiplier under transformations generated by the constraints (see e.g. [18] equation (3.26b) - note that the brackets of the constraints (B.2) and (B.3) involve the Gauss' law constraint [24]).

The transformation of the electric field will also be evaluated through its Poisson bracket with the Hamiltonian constraints and is

$$
\delta_{\xi} \pi^{k}=D_{m}\left(\xi F^{k m} \sqrt{g}\right)+\left(\xi^{m} \pi^{k}\right)_{, m}-\xi^{k},_{m} \pi^{m}
$$

where $D_{m}$ is the spatial covariant derivative. The transformation (B.6) coincides on-shell with the standard transformation.

To motivate the boundary conditions, consider first the zero-monopole sector. In Minkowski space, the electromagnetic potential for an electric charge at rest at the origin is given by

$$
A_{0} \sim \frac{e}{r}, A_{k}=0
$$

while the electromagnetic field behaves as

$$
F_{0 i} \sim \frac{e n^{i}}{r^{2}}, \quad F_{i j}=0
$$

where $n^{i}$ is the unit radial vector. By boosting this solution, one generates non-vanishing $r^{-1}$-order terms for $A_{k}$; these terms have the interesting property of being even under the parity $\mathbf{n} \rightarrow-\mathbf{n}$. Similarly, the leading-order term in the electromagnetic field has the property of being odd under the same parity transformation. These features remain valid if one superposes various charges.

This suggests taking as boundary conditions at spatial infinity (at each given time)

$$
A_{k}=\frac{a_{k}^{(1)}(\mathbf{n})}{r}+\frac{a_{k}^{(2)}(\mathbf{n})}{r^{2}}+o\left(r^{-2}\right)
$$

for the spatial components of the connexion and

$$
\pi^{k}=\frac{p_{(1)}^{k}(\mathbf{n})}{r^{2}}+\frac{p_{(2)}^{k}(\mathbf{n})}{r^{3}}+o\left(r^{-3}\right)
$$


for the electric field. These conditions imply

$$
F_{i k}=\frac{f_{i k}^{(1)}(\mathbf{n})}{r^{2}}+\frac{f_{i k}^{(2)}(\mathbf{n})}{r^{3}}+o\left(r^{-3}\right)
$$

for the magnetic field. We impose also that the first coefficients in $A_{k}$ and $\pi^{k}$ has definite parity properties:

$$
a_{k}^{(1)}(-\mathbf{n})=a_{k}^{(1)}(\mathbf{n}), \quad p_{(1)}^{k}(-\mathbf{n})=-p_{(1)}^{k}(\mathbf{n}) .
$$

It follows that

$$
f_{i k}^{(1)}(-\mathbf{n})=-f_{i k}^{(1)}(\mathbf{n})
$$

One easily verifies that these conditions are invariant under Lorentz transformations and futhermore guarantee the vanishing of the boundary term of the variational principle, namely $\delta A_{0} \pi^{i}+\delta A_{j} F^{j i}$, when integrated at infinity. In fact, the combined asymptotic conditions of [9, 14] and those given here are invariant under the full rigid $N=2$ SUSY algebra acting at infinity.

We shall verify for example the asymptotic Lorentz invariance of the above boundary conditions. The invariance of $(\mathrm{B.9})$ follows from $(\mathrm{B} .1)$ and $(\mathrm{B} .10),(\mathrm{B} .11)$ and the fact that for boosts and rotations, the leading orders of $\xi$ and $\xi^{k}$ are both parity-odd. Similarly, the invariance of (B.10) follows from $(\overline{B .6})$ and the same observation on the parity of the leading orders of $\xi$ and $\xi^{k}$. One should stress that the boundary conditions adopted here can probably be somewhat relaxed. However, our goal is not to provide here the most flexible admissible boundary behaviour, but to give a consistent and complete set of boundary conditions that enforce asymptotically the $N=2$ supersymmetry algebra.

The asymptotic symmetry algebra may actually involve also a central $U(1)$. This issue is somewhat subtle because there is no charged field in $\mathcal{N}_{4}=2$ (ungauged) supergravity. Whether there is a non trivial $U(1)$ at infinity depends on the topology of the spatial sections. To see this, one notes that the boundary conditions on the potential are invariant under gauge transformations that behave asymptotically as

$$
\delta_{\Lambda} A_{\mu}=\partial_{\mu} \Lambda
$$

with

$$
\Lambda=\lambda_{0}+\lambda(\mathbf{n})+o\left(r^{0}\right)
$$

where $\lambda_{0}$ is a constant and $\lambda(\mathbf{n})$ is parity-odd,

$$
\lambda(-\mathbf{n})=-\lambda(\mathbf{n}) .
$$


However, the transformations associated with $\lambda(\mathbf{n})$ are really irrelevant because their corresponding charges, given by the flux of the electric field times $\lambda(\mathbf{n})$ at infinity (up to non-written Gauss' law constraint terms)

$$
Q[\lambda(\mathbf{n})]=\int_{S_{\infty}} \lambda(\mathbf{n}) \boldsymbol{\pi} \cdot \mathbf{d} \mathbf{S}
$$

all vanish identically for the field configurations allowed here. Thus, one can factor them out. These transformations are somewhat the analogs of the supertranslations of the BMS group, which are eliminated in the same way through the parity conditions [9]. In fact, the main motivation for adopting similar parity conditions on the electromagnetic potential is to effectively remove the charges (B.17), which do not seem to have a direct physical interpretation (these charges are not associated with higher multipole moments).

Thus, only the constant piece $\lambda_{0}$ in $(\overline{B .15})$ is relevant. The charge associated with gauge transformation that tend to a constant at infinity reads (again up to unwritten Gauss' law terms)

$$
Q\left[\lambda_{0}\right]=\lambda_{0} \int_{S_{\infty}} \boldsymbol{\pi} \cdot \mathbf{d} \mathbf{S}
$$

There may be additional surface terms if there are other boundaries (other asymptotic regions, or other surfaces on which boundary conditions are required: horizons, surfaces surrounding singularities), but since we are interested here only in the asymptotic symmetry generators in a single asymptotic region (called "infinity"), we shall consider gauge transformations that are zero except in a vicinity of this asymptotic region. These gauge transformations are correctly generated by (B.18), without any other surface contribution. Being zero at the other boundaries (if any) they certainly preserve any set of specific boundary conditions given there.

Now, whether (B.18) identically vanishes or not depends on the topology of the spatial sections. If these are homeomorphic to $R^{3}$, then $(\mathrm{B} .18)$ is zero by Gauss' law and there is no non trivial $U(1)$ asymptotic generator. However, if the 2-sphere at infinity is not contractible, (B.18) needs not vanish ("charge without charge"). Furthermore, as a generator, (B.18) acts non trivially on the gauge-invariant Mandelstam variables

$$
M=\int_{\gamma} d x^{k} A_{k}
$$

where $\gamma$ is any path joining the asymptotic region being considered to any other asymptotic region. The variable $M$ is invariant under "proper" gauge 
transformations, i.e., under gauge transformations that vanish on all boundaries. In that sense, it is an observable. However, it is not invariant under global transformations characterized by a non-vanishing $\lambda_{0}$ provided it ends outside the component of infinity under consideration. One gets $\delta_{\Lambda} M=\lambda_{0}$, which implies that $M$ is conjugate to the electric charge measured at infinity,

$$
\left[M, \int_{S_{\infty}} \boldsymbol{\pi} \cdot \mathbf{d} \mathbf{S}\right]=1
$$

(again, we do not write explicitly the constraint terms that accompany the surface integral at infinity). There is thus a non-trivial $U(1)$ generator at infinity, which generates asymptotic symmetries.

Note that one would also find $U(1)$ symmetries on the other boundaries. The sum over all boundaries of the $U(1)$ charges is zero by Gauss' law (the flux lines can only end on the boundaries). The fact that the charges add up to zero just reflects the fact that the generator associated with everywhere constant gauge transformations vanishes, as it should (such transformations have no action on the fields).

The inclusion of magnetic charges is direct. Instead of imposing (B.9) on the potential $A_{k}$, one requires that it behaves asymptotically as

$$
A_{k}=A_{k}^{\text {mon }}+A_{k}^{\prime}
$$

where $A_{k}^{\text {mon }}$ is the potential for the magnetic monopole(s), living on a nontrivial $U(1)$ bundle over $S_{2}$ (and defined over patches), while $A_{k}^{\prime}$ is globally defined and subject to the same boundary conditions (B.9). The boundary conditions on the electric field are unchanged. The magnetic field has also the same asymptotic behaviour because the field of a monopole obeys (B.11). These more general boundary conditions are still Lorentz invariant because the variation of $A_{k}^{\text {mon }}$, which is globally defined, behaves as $A_{k}^{\prime}$. They are in fact also invariant under supersymmetry transformations and constant gauge transformations. Furthermore, the (graded) commutator of two local supersymmetry transformations that tend asymptotically to constants $\epsilon^{A}$ and $\zeta^{A}$ is the sum of a diffeomorphism that becomes asymptotically a translation with parameter $-i \kappa^{2} \bar{\epsilon}_{A} \gamma^{\mu} \zeta^{A}$ and a gauge transformation that behaves at infinity as a constant gauge transformation with parameter $\Phi=-i \kappa \bar{\epsilon}_{A} \varepsilon_{B}^{A} \zeta^{B}$ (the asymptotic value of the gauge transformation is defined up to a constant, but the ambiguity disappears if one imposes that $\Phi$ vanishes when $\epsilon$ or $\zeta$ is equal to zero). There is no sign of the magnetic charge in the asymptotic algebra of the asymptotic symmetry transformations. 
Let us turn now to the algebra of the charges defined, in the Lagrangian context, through the variations of the charges under the transformations generated by one another. The variation of the superpotential was given in equation (3.5), together with the result (3.4) for supergravity theories. Now in the special case where $\delta$ is another supersymmetry gauge transformation, that is $\delta:=\delta_{\zeta}$, the variation becomes:

$$
\begin{aligned}
\delta_{\zeta} U_{\bar{\epsilon}}^{\mu \nu} & =-i \bar{\epsilon}_{A} \gamma^{\mu \nu \sigma} \hat{\mathcal{D}}_{\sigma} \zeta^{A} \\
& =-i \bar{\epsilon}_{A} \gamma^{\mu \nu \sigma}\left(\mathcal{D}_{\sigma}+\frac{\kappa}{2} \varepsilon_{B}^{A}\left(\hat{F}_{\rho \sigma} \gamma^{\rho}+\tilde{\hat{F}}_{\rho \sigma} \gamma^{\rho} \gamma^{5}\right)\right) \zeta^{B} \\
& =-i \bar{\epsilon}_{A} \gamma^{\mu \nu \sigma} \mathcal{D}_{\sigma} \zeta^{A}-i \kappa \bar{\epsilon}_{A} \varepsilon_{B}^{A}\left(\hat{F}^{\mu \nu}+\gamma^{5} \tilde{\hat{F}}^{\mu \nu}\right) \zeta^{B}
\end{aligned}
$$

Where we used the $\left(\mathcal{N}_{4}=2\right)$ - "hatted" covariant derivative defined in appendix A, after equation (A.2).

Let us now integrate (B.22) at spatial infinity:

1. The first term in the rhs of $(\mathrm{B} .22)$ is nothing but the Nester-Witten superpotential [25], and so the covariant version of ADM mass (and momentum). We will denote it by $H_{\xi}$, where $\xi^{\mu}$ is an asymptotic displacement and is given by $\xi^{\mu}=-i \kappa^{2} \bar{\epsilon}_{A} \gamma^{\mu} \zeta^{A}$. The idea of relating the positivity of gravitational mass and local supersymmetry can be found in 26]. The supergravity origin and positivity of the NesterWitten superpotential [25] was already established for the $\mathcal{N}_{4}=1$ supergravity in [27].

2. The second term gives electric and magnetic contributions ("central" charges) to the algebra of supersymmetry. Due to the asymptotic behavior of the gravitino (falling off like $1 / r^{2}$ ), the hatted field strength can be replaced by the ordinary field strength, the difference between both falling off like $1 / r^{4}$.

Let us define:

$$
Q:=-\int_{S_{\infty}}\left(i \kappa \bar{\epsilon}_{A} \varepsilon_{B}^{A} \zeta^{B}\right) F^{0 i} \quad \text { and } \quad P:=-\int_{S_{\infty}}\left(i \kappa \bar{\epsilon}_{A} \varepsilon_{B}^{A} \gamma^{5} \zeta^{B}\right) \tilde{F}^{0 i}
$$

Then we find that the algebra of the SUSY charges (which generate the asymptotic supersymmetric invariance of $\mathcal{N}_{4}=2$ supergravity) has a magnetic central charge besides the expected $U(1)$ generator. These are familiar from rigid supersymmetry. Explicitly, we find the algebra

$$
\delta_{\zeta} Q_{\bar{\epsilon}}:=\left[Q_{\bar{\epsilon}}, Q_{\zeta}\right]=H_{\xi}+Q+P
$$


in agreement with [14, 19]. The electric and magnetic charges appear as central charges for the $N=2$ supersymmetry algebra. There is, however, an apparent difference between the two in the present (electric) formulation of $\mathcal{N}_{4}=2$ supergravity. While the Noetherian electric charge is a non trivial generator which does act on some canonical fields (when it does not vanish identically), the magnetic charge commutes with all of them and not just with the other generators of the asymptotic symmetry algebra, even when it is non zero. It is (in this limited treatment) a fully central charge.

It is customary to adjust the constants in the generators of the asymptotic symmetries so that these vanish on some specified background. When specialized to that background, (B.23) becomes

$$
\delta_{\zeta} Q_{\bar{\epsilon}}=P \text { on background }
$$

$\left(H_{\xi}=0, Q=0\right)$. The central charge is the variation of the background generator of asymptotic supersymmetries. The magnetic central charge can (and does) arise because the electromagnetic field on a non-trivial $U(1)$ bundle is non zero and breaks some of the supersymmetries $\left(\delta_{\zeta} Q_{\bar{\epsilon}} \neq 0\right)$.

Note that in a dual (magnetic) formulation the role of electric and magnetic charges would be exchanged, a fully duality invariant treatment should restore the symmetry between the two.

The algebra of the asymptotic symmetry transformations (once more defined on the electric canonical variables) and the algebra of their generators are different. The algebra (B.23) gives another example of the general theorem of [29] which establishes that extra central charges are allowed in the asymptotic charge algebras. This has been already used in [20] and generalises the classical ambiguity of the charge algebra relative to the symmetry algebra in Hamiltonian dynamics.

\section{References}

[1] E. Noether, Nachr. Ges. Wiss. zu Goettingen (1918) 235.

[2] P.G. Bergmann, Phys. Rev. 75 (1949) 680

J. Heller, Phys. Rev. 81 (1951) 946.

[3] J.G. Fletcher, Rev. Mod. Phys. 32 (1960) 65.

[4] P.G. Bergmann, in Handbuch der Physik 4 (1962) 237. 
[5] B. Julia, in Proceedings of the 4th Johns Hopkins Workshop on Current problems in Particle theory, Bonn (1980) ed. R. Casalbuoni et al.

[6] D. Bak, D. Cangemi and R. Jackiw, Phys. Rev. D 49 (1994) 5173.

[7] B. Julia and S. Silva, Class. Qu. Grav., 15 (1998) 2173, gr-qc/9804029.

[8] R. Arnowitt, S. Deser and C.W. Misner, Phys Rev. 122 (1961) 3.

[9] T. Regge and C. Teitelboim, Ann. of Phys. 88 (1974) 286.

[10] A. Ashtekar, in "General Relativity and Gravitation" (vol.2), ed. A. Held (Plenum Press, New York), and references therein.

[11] L.F. Abbott and S. Deser, Phys. Let. B 116 (1982) 259.

[12] S. Silva, Nucl. Phys. B558 (1999) 391, hep-th/9809109.

[13] G. Barnich, F. Brandt and M. Henneaux, Commun. Math. Phys. 174 (1995) 57.

[14] C. Teitelboim Phys. Lett. 69B (1977) 240.

[15] A. Komar, Phys. Rev. 113 (1959) 934.

[16] J. Katz, Class. Quantum Grav. 2 (1985) 423; J. Katz, J. Bičák, D. Lynden-Bell, Phys. Rev. D 55 (1997).

[17] M. Henneaux and C. Teitelboim, Commun. Math. Phys. 98 (1985) 391.

[18] M. Henneaux and C. Teitelboim, Quantization of Gauge Systems, Princeton University Press, Princeton 1992.

[19] G.W. Gibbons and C.M. Hull, Phys. Lett. 109B (1982) 190; C.M. Hull, Nucl. Phys. B509 (1998) 216, hep-th/9705162.

[20] J.D. Brown and M. Henneaux, Commun. Math. Phys. 104 (1986) 207.

[21] B. Julia and S. Silva, in preparation.

[22] B. Julia and S. Silva First order formulations of supergravities, in preparation.

[23] S. Deser and B. Zumino, Phys. Lett. 62B 3 (1976) 335. 
[24] C. Teitelboim, The Hamiltonian structure of spacetime, in "General Relativity and Gravitation" (vol.1), ed. A. Held, pp. 195-225 (Plenum Press, New York).

[25] J.N. Nester, Phys. Lett. 83 A (1981) 241; E. Witten, Com. Math. Phys. 80 (1981) 381.

[26] S. Deser and C. Teitelboim, Phys. Rev. Lett. 39 (1977) 249; M.T. Grisaru, Phys. Lett. 73 B (1978) 207.

[27] G.T. Horowitz, A. Strominger, Phys. Rev. D 12 (1983) 2793; C.M. Hull, Com. Math. Phys. 90 (1983) 545.

[28] A.H. Taub, Lett. Math. Phys. 9 (1985) 243, J. Geom. Phys. 23 (1985) 61; J. Lee and D. Sorkin, Com. Math. Phys. 116 (1988) 353.

[29] J.D. Brown and M. Henneaux, J. Math. Phys., 27 (2) (1986) 489. 